\title{
Pulsed EPR and DFT Characterization of Radicals Produced by Photo-Oxidation of
}

Zeaxanthin and Violaxanthin on Silica-Alumina

A. Ligia Focsan, ${ }^{\dagger}$ Tatyana A. Konovalova, ${ }^{\dagger}$ Michael K. Bowman, ${ }^{\dagger}$ David A. Dixon, ${ }^{\dagger}$ Lowell D. Kispert, ${ }^{\dagger}$ Péter Molnár, ${ }^{\ddagger}$ and Jozsef Deli ${ }^{\ddagger}$

${ }^{\dagger}$ Department of Chemistry, University of Alabama, Tuscaloosa, AL 35487-0336, USA

${ }^{\ddagger}$ Department of Medical Chemistry, University of Pẻcs, H-7601, Pẻcs, Hungary

Supporting Information: Echo-induced field-swept EPR spectrum of zeaxanthin radicals generated by irradiation at $77 \mathrm{~K}$ on silica-alumina, Fourier transform of the 2-pulse ESEEM spectrum of zeaxanthin radicals on silica-alumina, Mims ENDOR spectra of zeaxanthin and violaxanthin radicals on silica-alumina, Optimized x, y, z coordinates at B3LYP/6-31G (d, p) level for Zea ${ }^{\bullet+}, \# Z e a^{\bullet}(4), \# Z e a^{\bullet}(5), \# Z e a^{\bullet}(9), \# Z e a^{\bullet}(13), \mathrm{Vio}^{\bullet+}, \# V_{i o}^{\bullet}(4), \# V_{i o}(5), \# V^{\bullet}(9)$ and \#Vio ${ }^{\circ}(13)$. 


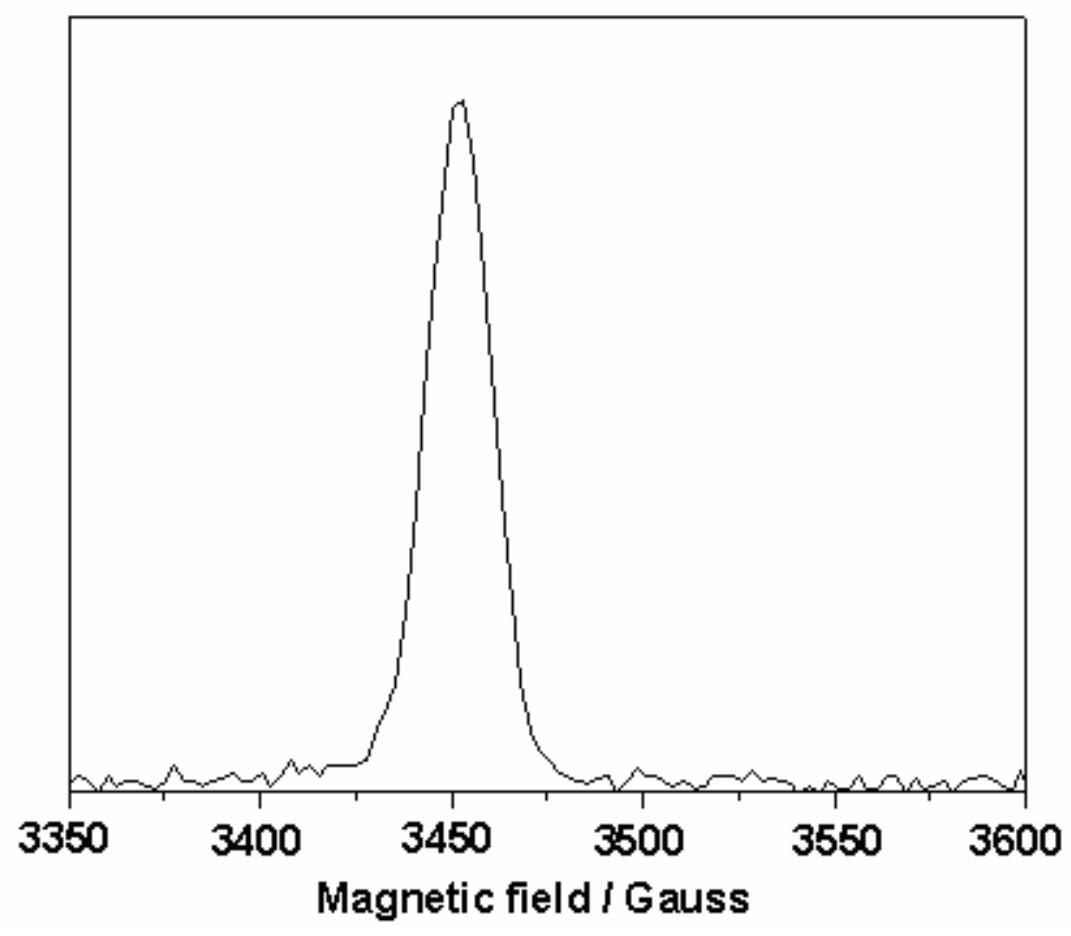

Figure S1. Echo-induced field-swept EPR spectrum of zeaxanthin radicals generated by irradiation at $77 \mathrm{~K}$ on silica-alumina. Experimental conditions: $\tau=200 \mathrm{~ns}$; repetition time (SRT) $=1000 \mu \mathrm{s} ;$ microwave frequency $=9.671 \mathrm{GHz} ; \mathrm{T}=50 \mathrm{~K}$. 


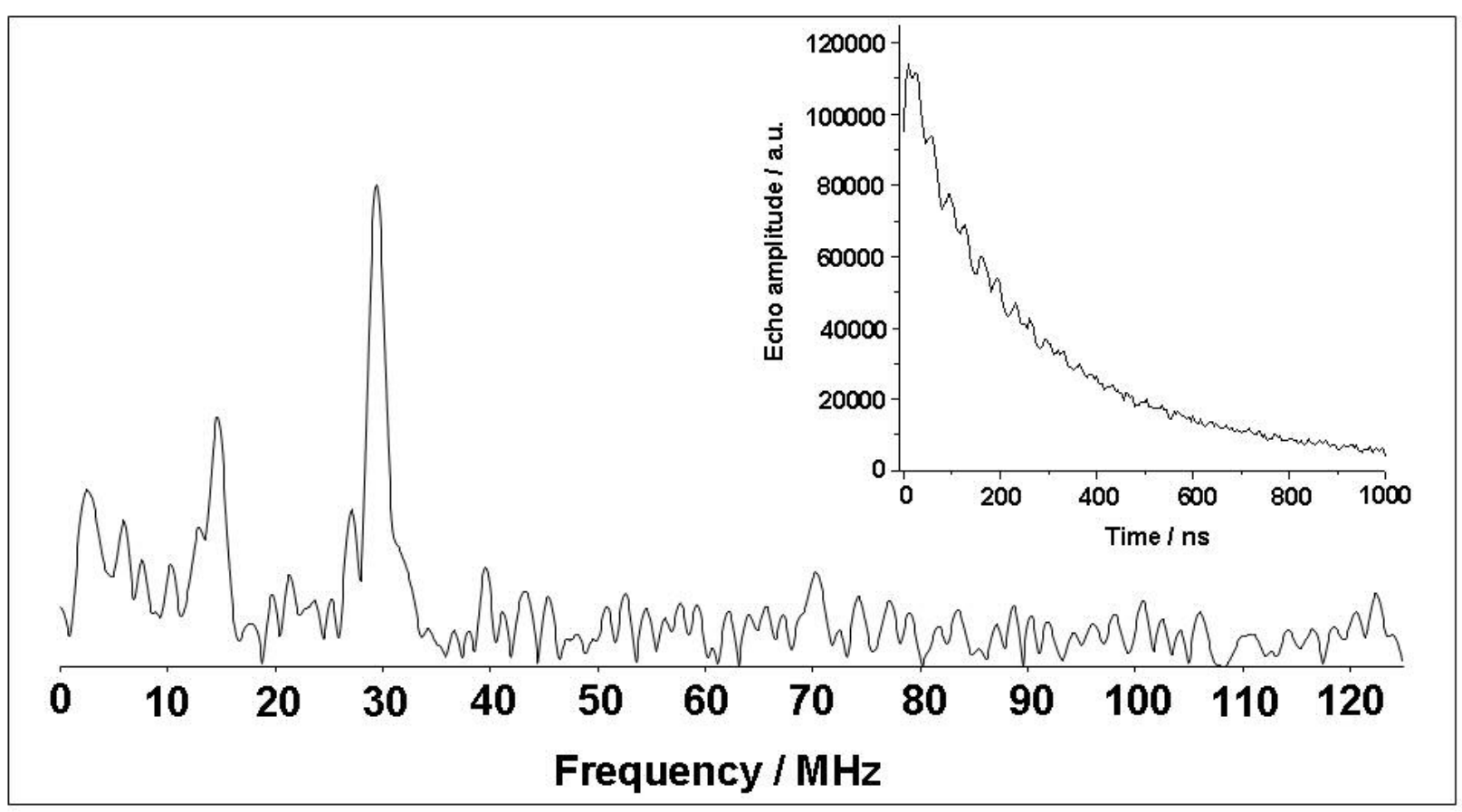

Figure S2. Fourier transform of the 2-pulse ESEEM spectrum of zeaxanthin radicals on silicaalumina. Parameters: $\mathrm{mw}$ frequency $=9.672 \mathrm{GHz}, \mathrm{B}=3450 \mathrm{G}$, repetition time $=1023.06 \mu \mathrm{s}, \mathrm{T}=$ $50 \mathrm{~K}$. 


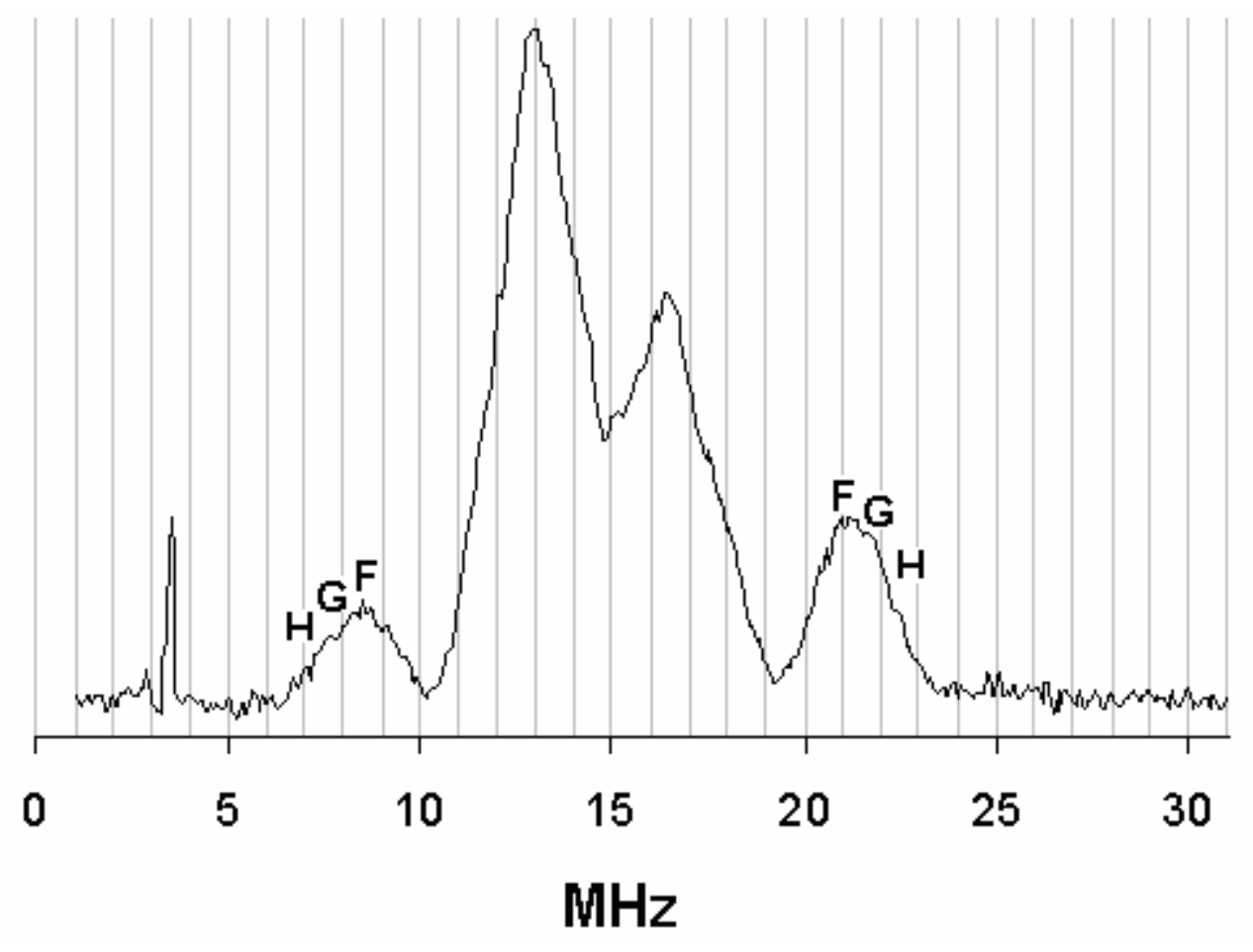

Figure S3. Mims ENDOR spectrum of zeaxanthin radicals on silica-alumina. Parameters: $\mathrm{T}=40$ $\mathrm{K}, \mathrm{B}=3460 \mathrm{G}, \nu=9.691228 \mathrm{GHz}, \tau=108 \mathrm{~ns}, \mathrm{MW} \pi$ pulse $=32 \mathrm{~ns}, \mathrm{RF} \pi$ pulse $=10 \mu \mathrm{s}$, repetition time $=1021.02 \mu \mathrm{s}$. 


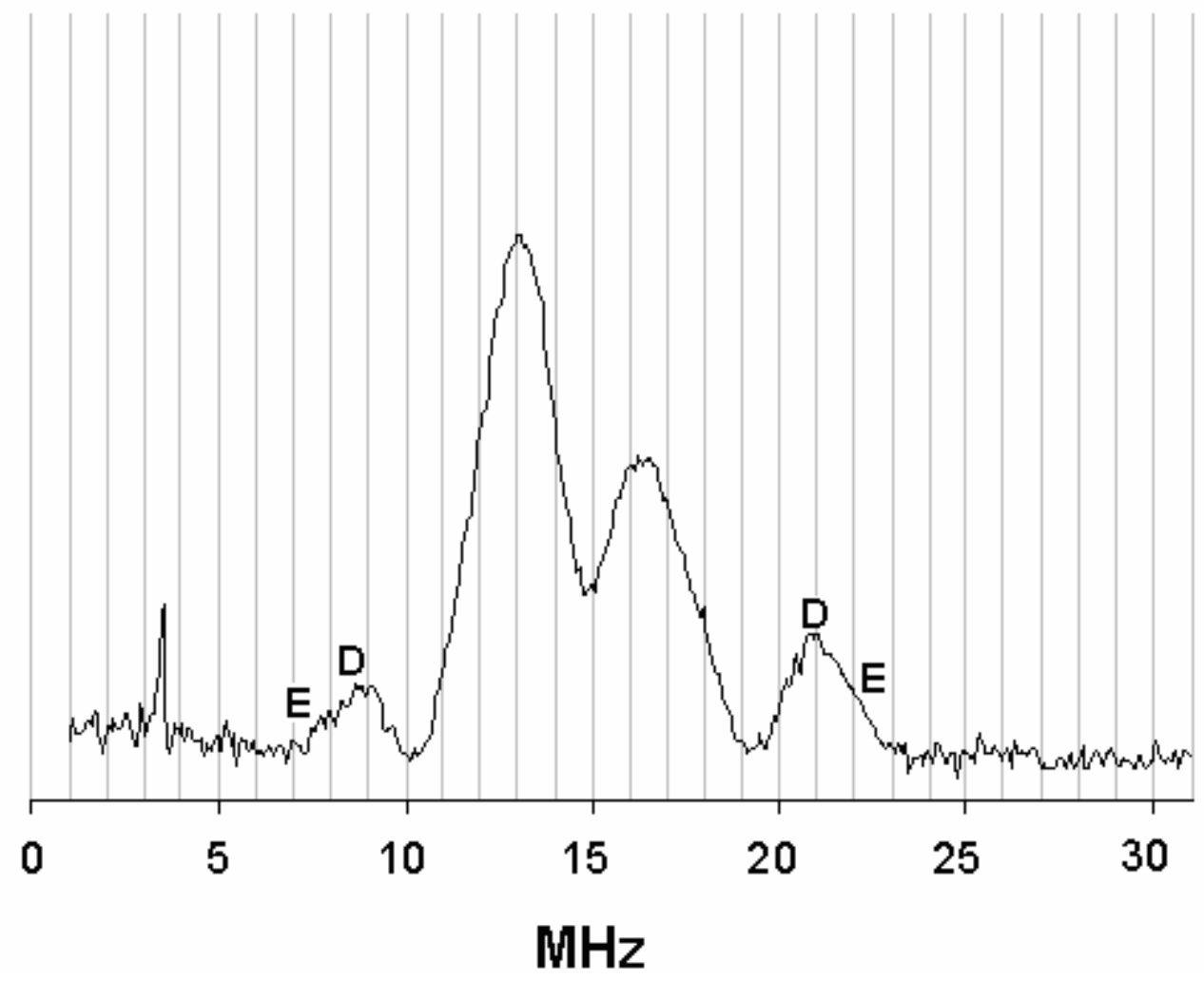

Figure S4. Mims ENDOR spectrum of violaxanthin radicals on silica-alumina. Parameters: $\mathrm{T}=$ $40 \mathrm{~K}, \mathrm{~B}=3460 \mathrm{G}, v=9.687040 \mathrm{GHz}, \tau=108 \mathrm{~ns}, \mathrm{MW} \pi$ pulse $=32 \mathrm{~ns}, \mathrm{RF} \pi$ pulse $=10 \mu \mathrm{s}$, repetition time $=1021.02 \mu \mathrm{s}$. 
TABLE S1: Optimized $\mathrm{x}, \mathrm{y}, \mathrm{z}$ coordinates at B3LYP/6-31G $(\mathrm{d}, \mathrm{p})$ level for Zea ${ }^{\bullet+}$.

$\begin{array}{cccc}\mathrm{C} & 11.619217 & 0.073290 & -0.189794 \\ \mathrm{C} & 11.695337 & -1.049653 & -0.967501 \\ \mathrm{C} & 12.824054 & 0.583996 & 0.643733 \\ \mathrm{C} & 14.109734 & -0.209449 & 0.310784 \\ \mathrm{H} & 14.858001 & -0.010562 & 1.089038 \\ \mathrm{H} & 14.535988 & 0.148235 & -0.635035 \\ \mathrm{C} & 13.875186 & -1.708880 & 0.167874 \\ \mathrm{H} & 13.432523 & -2.108743 & 1.094133 \\ \mathrm{C} & 12.912247 & -1.941116 & -0.984610 \\ \mathrm{H} & 13.463820 & -1.788262 & -1.924941 \\ \mathrm{H} & 12.590628 & -2.990511 & -1.006787 \\ \mathrm{C} & 13.122064 & 2.072174 & 0.338396 \\ \mathrm{H} & 12.352729 & 2.749033 & 0.721786 \\ \mathrm{H} & 14.062111 & 2.360169 & 0.819903 \\ \mathrm{H} & 13.230448 & 2.244988 & -0.737069 \\ \mathrm{C} & 12.490026 & 0.447637 & 2.149386 \\ \mathrm{H} & 13.308120 & 0.844053 & 2.760524 \\ \mathrm{H} & 11.582509 & 1.004743 & 2.404196 \\ \mathrm{H} & 12.324757 & -0.594900 & 2.437338 \\ \mathrm{C} & 10.634255 & -1.504017 & -1.939079 \\ \mathrm{H} & 10.048510 & -2.337360 & -1.528019 \\ \mathrm{H} & 9.942487 & -0.711916 & -2.226886 \\ \mathrm{H} & 11.114321 & -1.890081 & -2.845619 \\ \mathrm{C} & 10.415553 & 0.886457 & -0.091294 \\ \mathrm{H} & 10.579040 & 1.946053 & 0.083503 \\ \mathrm{C} & 9.115200 & 0.458382 & -0.108068 \\ \mathrm{H} & 8.914883 & -0.606878 & -0.170820 \\ \mathrm{C} & 7.961789 & 1.305389 & -0.009934 \\ \mathrm{C} & 6.713348 & 0.692404 & -0.001681 \\ \mathrm{H} & 6.707755 & -0.395513 & -0.056463 \\ \mathrm{C} & 5.451297 & 1.313534 & 0.059807 \\ \mathrm{H} & 5.401392 & 2.397007 & 0.103246 \\ \mathrm{C} & 4.262832 & 0.596845 & 0.058551 \\ \mathrm{H} & 4.336564 & -0.488960 & 0.018698 \\ \mathrm{C} & 2.960562 & 1.143757 & 0.098044 \\ \mathrm{C} & 1.881137 & 0.247745 & 0.084254 \\ \mathrm{H} & 2.137760 & -0.810575 & 0.053644 \\ \mathrm{C} & 0.517798 & 0.551541 & 0.099618 \\ \mathrm{H} & 0.198740 & 1.590578 & 0.123616 \\ & 8.138344 & 2.800421 & 0.072169 \\ \mathrm{H} & 7.192701 & 3.335818 & 0.147607 \\ \mathrm{H} & 8.667295 & 3.177648 & -0.809252\end{array}$




\begin{tabular}{|c|c|c|c|}
\hline $\mathrm{C}$ & 2.771687 & 2.640760 & 46998 \\
\hline & 3.264466 & 3.065344 & 1.028058 \\
\hline & 3.213830 & 3.117069 & -0.734640 \\
\hline & 1.723128 & 2.933202 & 0.186564 \\
\hline & -0.479103 & -0.425764 & 0.080353 \\
\hline & -0.158407 & -1.464367 & 0.058995 \\
\hline & -1.843419 & -0.125420 & 0.082035 \\
\hline & -2.104066 & 0.932222 & 0.097233 \\
\hline & -2.918437 & -1.026106 & 0351 \\
\hline & -4.224680 & -0.485557 & 0.04 \\
\hline $\mathrm{H}$ & -4.305461 & 0.600422 & 485 \\
\hline & -5.406846 & -1.210524 & 0.017454 \\
\hline $\mathrm{H}$ & -5.347501 & -2.294344 & 522 \\
\hline & -6.676276 & -0.599941 & -0.011301 \\
\hline 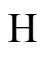 & -6.6 & 312 & -0. \\
\hline$C$ & -7.916098 & -1.226733 & 228 \\
\hline 0 & -9.083217 & -0.390727 & -0 \\
\hline $\mathrm{H}$ & -8.89 & 577 & -0 . \\
\hline C & -2.721999 & -2.522861 & 0.044602 \\
\hline $\mathrm{H}$ & -3.14 & 0504 & -0.8 \\
\hline $\mathrm{H}$ & -1.672862 & -2.812160 & 0.090600 \\
\hline $\mathrm{H}$ & -3.228326 & -2.989452 & 0.896083 \\
\hline $\mathrm{C}$ & -10.375428 & -0.836951 & -0.079658 \\
\hline $\mathrm{H}$ & -10.525223 & -1.908043 & \\
\hline $\mathrm{C}$ & -11.598833 & -0.044529 & 774 \\
\hline $\mathrm{C}$ & -11.746804 & 1.072272 & -0.8 \\
\hline $\mathrm{C}$ & -13.022511 & 1.887967 & -0.8 \\
\hline $\mathrm{H}$ & -13.220642 & 2.261270 & -1.8 \\
\hline $\mathrm{H}$ & -12.865032 & 2.783747 & -0.259488 \\
\hline S & -14.248067 & 1.156718 & -0.331055 \\
\hline $\mathrm{H}$ & -14.595505 & 0.426399 & -1.078122 \\
\hline $\mathrm{C}$ & -13.865370 & 0.434308 & 0.948250 \\
\hline $\mathrm{H}$ & & -0.044896 & 042 \\
\hline $\mathrm{H}$ & -13.532530 & 1.189852 & 1.671200 \\
\hline$C$ & -12.754480 & -0.619368 & 0.745862 \\
\hline 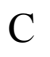 & -8.075297 & -2.725720 & -0.035717 \\
\hline $\mathrm{H}$ & -8.612105 & -3.062981 & -0.928541 \\
\hline $\mathrm{H}$ & -7.123418 & -3.254163 & -0.000502 \\
\hline $\mathrm{H}$ & -8.661946 & -3.045709 & 0.832545 \\
\hline 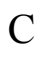 & -10.699312 & 1.610486 & -1.830704 \\
\hline $\mathrm{H}$ & -10.202174 & 2.493677 & -1.406146 \\
\hline $\mathrm{H}$ & -9.929687 & 0.881482 & -2.084397 \\
\hline $\mathrm{H}$ & -11.172915 & 1.945917 & -2.759703 \\
\hline $\mathrm{C}$ & -13.337579 & -1.879440 & 0.058677 \\
\hline $\mathrm{H}$ & -12.581357 & -2.660002 & -0.074864 \\
\hline 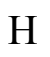 & -14.137212 & -2.305814 & 0.673499 \\
\hline
\end{tabular}




$\begin{array}{rrrr}\mathrm{H} & -13.751535 & -1.652863 & -0.927694 \\ \mathrm{C} & -12.240537 & -1.014047 & 2.150203 \\ \mathrm{H} & -11.759585 & -0.165991 & 2.647777 \\ \mathrm{H} & -13.078865 & -1.339496 & 2.774908 \\ \mathrm{H} & -11.520615 & -1.837454 & 2.115759 \\ \mathrm{O} & -15.298039 & 2.062194 & -0.008817 \\ \mathrm{H} & -15.618028 & 2.461434 & -0.828629 \\ \mathrm{O} & 15.067555 & -2.417454 & -0.151897 \\ \mathrm{H} & 15.679715 & -2.329884 & 0.590695\end{array}$

TABLE S2: Optimized x, y, z coordinates at B3LYP/6-31G (d, p) level for \#Zea (4).

$\begin{array}{lrrr}\mathrm{C} & -11.618251 & 0.042314 & 0.214815 \\ \mathrm{C} & -11.718889 & -1.040235 & 1.028445 \\ \mathrm{C} & -12.735333 & 0.432755 & -0.783128 \\ \mathrm{C} & -14.012660 & -0.405093 & -0.542825 \\ \mathrm{H} & -14.683469 & -0.288073 & -1.405659 \\ \mathrm{H} & -14.552762 & -0.020155 & 0.331826 \\ \mathrm{C} & -13.723467 & -1.879859 & -0.290131 \\ \mathrm{H} & -13.162499 & -2.299156 & -1.140751 \\ \mathrm{C} & -12.882015 & -2.007447 & 0.968507 \\ \mathrm{H} & -13.541458 & -1.852458 & 1.836196 \\ \mathrm{H} & -12.508902 & -3.035576 & 1.065848 \\ \mathrm{C} & -13.129843 & 1.920392 & -0.619167 \\ \mathrm{H} & -12.337062 & 2.600801 & -0.942399 \\ \mathrm{H} & -14.010879 & 2.138159 & -1.233081 \\ \mathrm{H} & -13.379946 & 2.152668 & 0.421367 \\ \mathrm{C} & -12.225083 & 0.223591 & -2.229650 \\ \mathrm{H} & -12.978006 & 0.552024 & -2.955879 \\ \mathrm{H} & -11.310481 & 0.797444 & -2.406547 \\ \mathrm{H} & -11.994696 & -0.827210 & -2.429846 \\ \mathrm{C} & -10.732852 & -1.390179 & 2.117760 \\ \mathrm{H} & -10.112791 & -2.253178 & 1.839496 \\ \mathrm{H} & -10.065613 & -0.565110 & 2.367772 \\ \mathrm{H} & -11.277041 & -1.686275 & 3.023977 \\ \mathrm{C} & -10.440314 & 0.928228 & 0.211999 \\ \mathrm{H} & -10.640225 & 1.995969 & 0.169314 \\ \mathrm{C} & -9.144839 & 0.525138 & 0.192591 \\ \mathrm{H} & -8.939926 & -0.543538 & 0.163315 \\ \mathrm{C} & -7.976574 & 1.383963 & 0.186688 \\ \mathrm{C} & -6.737141 & 0.793453 & 0.135146 \\ \mathrm{H} & -6.720818 & -0.296248 & 0.095508 \\ \mathrm{C} & -5.456064 & 1.421826 & 0.130905 \\ \mathrm{H} & -5.410821 & 2.505715 & 0.181888 \\ \mathrm{C} & -4.279301 & 0.715317 & 0.068197\end{array}$




\begin{tabular}{|c|c|c|c|}
\hline & & & \\
\hline & & & \\
\hline & & & \\
\hline & & & \\
\hline & -0.50 & & \\
\hline & & & \\
\hline & -8.179 & & \\
\hline & -7.2 & & \\
\hline & -8.7 & & \\
\hline & -8.7 & & 259 \\
\hline & -2.7 & & \\
\hline & -3.2 & & 23 \\
\hline & -3.2 & & \\
\hline & -1.7 & & \\
\hline & 0.4 & & \\
\hline & 0.1 & & -0 \\
\hline & 1.8 & -0 . & \\
\hline $\mathrm{H}$ & 2.1 & & \\
\hline & 2.9 & -0.8 & \\
\hline & 4.2 & & \\
\hline $\mathrm{H}$ & 4.3 & & \\
\hline & 5.4 & & \\
\hline $\mathrm{H}$ & 5.3 & & \\
\hline & & -0. & \\
\hline $\mathrm{H}$ & & & \\
\hline & $7.9^{\prime}$ & -1. & \\
\hline 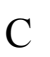 & & & \\
\hline $\mathrm{H}$ & 8.8 & & \\
\hline O & 2.7 & -2. & \\
\hline 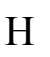 & 3.1 & -2.9 & \\
\hline-7 & 1.6 & -2.6 & \\
\hline $\mathrm{H}$ & 3.21 & -2.7 & \\
\hline O & & & \\
\hline $\mathrm{H}$ & & & 365 \\
\hline $\mathrm{C}$ & & -0 . & \\
\hline $\mathrm{C}$ & 11.8 & & 63 \\
\hline O & & & \\
\hline $\mathrm{H}$ & 13. & 2.8 & 359 \\
\hline $\mathrm{C}$ & & & \\
\hline $\mathrm{H}$ & 14.9 & & 086 \\
\hline $\mathrm{C}$ & 13.9 & 853 & -0 \\
\hline $\mathrm{H}$ & & -0.5 & \\
\hline $\mathrm{H}$ & 13.5 & 417 & 318 \\
\hline $\mathrm{C}$ & & -0.933573 & -0.077970 \\
\hline $\mathrm{C}$ & 8.110 & -2.621814 & -0.336514 \\
\hline 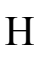 & 8.618794 & -3.084314 & 0.516864 \\
\hline
\end{tabular}




$\begin{array}{cccc}\mathrm{H} & 7.146663 & -3.117045 & -0.450817 \\ \mathrm{H} & 8.702276 & -2.854010 & -1.229191 \\ \mathrm{C} & 10.678544 & 2.070614 & 1.140589 \\ \mathrm{H} & 9.988077 & 2.422103 & 0.365987 \\ \mathrm{H} & 10.096153 & 1.490831 & 1.862546 \\ \mathrm{H} & 11.066126 & 2.956520 & 1.650380 \\ \mathrm{C} & 13.506923 & -1.364027 & 1.305816 \\ \mathrm{H} & 12.818568 & -2.067750 & 1.784777 \\ \mathrm{H} & 14.478477 & -1.860538 & 1.198007 \\ \mathrm{H} & 13.630471 & -0.513044 & 1.980562 \\ \mathrm{C} & 12.785510 & -2.201712 & -0.937147 \\ \mathrm{H} & 12.322705 & -1.975868 & -1.903020 \\ \mathrm{H} & 13.768778 & -2.643816 & -1.129400 \\ \mathrm{H} & 12.185494 & -2.969348 & -0.439089 \\ \mathrm{O} & 14.953921 & 2.128838 & -0.979388 \\ \mathrm{H} & 15.223682 & 2.918776 & -0.492285 \\ \mathrm{O} & -14.916096 & -2.636562 & -0.076915 \\ \mathrm{H} & -15.448609 & -2.575576 & -0.880995\end{array}$

TABLE S3: Optimized x, y, z coordinates at B3LYP/6-31G (d, p) level for \#Zea (5).

$\begin{array}{rrrr}\mathrm{C} & -11.565740 & 0.053203 & 0.266416 \\ \mathrm{C} & -11.664105 & -0.990705 & 1.129666 \\ \mathrm{C} & -12.686425 & 0.396718 & -0.744824 \\ \mathrm{C} & -13.963592 & -0.427264 & -0.459994 \\ \mathrm{H} & -14.637750 & -0.350468 & -1.324731 \\ \mathrm{H} & -14.499497 & -0.000475 & 0.397649 \\ \mathrm{C} & -13.675356 & -1.888804 & -0.138820 \\ \mathrm{H} & -13.118680 & -2.348869 & -0.970905 \\ \mathrm{C} & -12.828689 & -1.957874 & 1.120670 \\ \mathrm{H} & -13.484037 & -1.760084 & 1.982766 \\ \mathrm{H} & -12.456905 & -2.980857 & 1.265554 \\ \mathrm{C} & -13.079614 & 1.890729 & -0.651961 \\ \mathrm{H} & -12.288403 & 2.554118 & -1.012077 \\ \mathrm{H} & -13.963484 & 2.078346 & -1.271713 \\ \mathrm{H} & -13.324836 & 2.173796 & 0.377075 \\ \mathrm{C} & -12.181591 & 0.117050 & -2.181457 \\ \mathrm{H} & -12.936182 & 0.412285 & -2.920145 \\ \mathrm{H} & -11.265994 & 0.679010 & -2.388894 \\ \mathrm{H} & -11.954748 & -0.942844 & -2.332055 \\ \mathrm{C} & -10.673723 & -1.290868 & 2.229855 \\ \mathrm{H} & -10.056123 & -2.166847 & 1.989266 \\ \mathrm{H} & -10.004307 & -0.456141 & 2.438726 \\ \mathrm{H} & -11.214254 & -1.543766 & 3.151241 \\ \mathrm{C} & -10.388390 & 0.937797 & 0.217911\end{array}$




\begin{tabular}{|c|c|c|c|}
\hline $\mathrm{H}$ & -10.589120 & 2.001840 & 0.121336 \\
\hline $\mathrm{C}$ & -9.090876 & 0.536549 & 0.215094 \\
\hline & -8.883514 & -0.531802 & 0.237425 \\
\hline & -7.927117 & 1.396385 & 0.161826 \\
\hline & -6.682757 & 0.807371 & 0.131466 \\
\hline $\mathrm{H}$ & -6.663762 & -0.282998 & 0.144194 \\
\hline & -5.408207 & 1.438053 & 0.088476 \\
\hline I & -5.365920 & 2.523218 & 0.085450 \\
\hline & -4.223616 & 0.732142 & 0.052537 \\
\hline $\mathrm{H}$ & -4.293148 & -0.355974 & 0.051434 \\
\hline & -2.911102 & 1.276440 & 0.016983 \\
\hline & -1.826485 & 0.393599 & -0.024971 \\
\hline & -2.078643 & -0.667462 & -0.032621 \\
\hline & -0.461771 & 0.696187 & -0.058477 \\
\hline & -0.144266 & 1.736839 & -0.047013 \\
\hline$C$ & -8.133059 & 2.890653 & 0.141911 \\
\hline $\mathrm{H}$ & -7.194303 & 3.444355 & 0.115085 \\
\hline $\mathrm{H}$ & -8.720456 & 3.191988 & -0.734107 \\
\hline $\mathrm{H}$ & -8.691006 & 3.220372 & 1.026212 \\
\hline & -2.734320 & 2.776380 & 0.025546 \\
\hline $\mathrm{H}$ & -3.229618 & 3.234090 & -0.838908 \\
\hline & -3.183406 & 3.218686 & 0.922544 \\
\hline $\mathrm{H}$ & -1.686496 & 3.076155 & 0.000154 \\
\hline & 0.548100 & -0.277033 & -0.106755 \\
\hline $\mathrm{H}$ & 0.231518 & -1.318723 & -0.122548 \\
\hline & 1.905400 & 0.011736 & -0.134391 \\
\hline $\mathrm{H}$ & 2.188507 & 1.064159 & -0.111934 \\
\hline & 2.971911 & -0.926096 & -0.187841 \\
\hline 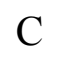 & 4.274917 & -0.445315 & -0.197239 \\
\hline $\mathrm{H}$ & 4.387202 & 0.638623 & -0.160670 \\
\hline & 5.485176 & -1.184612 & -0.246063 \\
\hline $\mathrm{H}$ & 5.427950 & -2.268463 & -0.287689 \\
\hline C & 6.725498 & -0.592945 & -0.239251 \\
\hline $\mathrm{H}$ & 6.761594 & 0.495547 & -0.192449 \\
\hline $\mathrm{C}$ & 7.997294 & -1.261723 & -0.283053 \\
\hline $\mathrm{C}$ & 9.136741 & -0.491595 & -0.255495 \\
\hline & 8.994596 & 0.585633 & -0.223162 \\
\hline 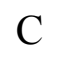 & 2.649366 & -2.403023 & -0.229352 \\
\hline & 3.541955 & -3.025264 & -0.295473 \\
\hline 11 & 2.098751 & -2.709395 & 0.667582 \\
\hline & 2.016111 & -2.641011 & -1.091467 \\
\hline 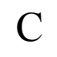 & 10.498146 & -0.949805 & -0.233031 \\
\hline & 10.642957 & -2.022146 & -0.140646 \\
\hline & 11.624220 & -0.179711 & -0.255649 \\
\hline & 11.597888 & 1.291231 & -0.445912 \\
\hline & 12.419676 & 2.116801 & 0.537438 \\
\hline
\end{tabular}




$\begin{array}{cccc}\mathrm{H} & 11.755025 & 2.563966 & 1.290327 \\ \mathrm{H} & 12.914032 & 2.941494 & 0.011718 \\ \mathrm{C} & 13.513366 & 1.282344 & 1.225485 \\ \mathrm{H} & 13.074409 & 0.733124 & 2.074108 \\ \mathrm{C} & 14.072954 & 0.279444 & 0.225754 \\ \mathrm{H} & 14.974279 & -0.188152 & 0.634742 \\ \mathrm{H} & 14.380191 & 0.830167 & -0.672407 \\ \mathrm{C} & 13.029315 & -0.814157 & -0.137786 \\ \mathrm{C} & 8.013321 & -2.771174 & -0.362297 \\ \mathrm{H} & 9.022063 & -3.179429 & -0.423922 \\ \mathrm{H} & 7.525716 & -3.215357 & 0.513456 \\ \mathrm{H} & 7.464307 & -3.119433 & -1.244776 \\ \mathrm{C} & 10.944009 & 1.874989 & -1.462555 \\ \mathrm{H} & 10.385453 & 1.293285 & -2.187863 \\ \mathrm{H} & 10.963824 & 2.952730 & -1.602986 \\ \mathrm{C} & 13.077038 & -1.927687 & 0.932193 \\ \mathrm{H} & 12.397061 & -2.751802 & 0.699586 \\ \mathrm{H} & 14.087158 & -2.347444 & 0.988391 \\ \mathrm{H} & 12.811404 & -1.549522 & 1.924139 \\ \mathrm{C} & 13.413047 & -1.420877 & -1.508280 \\ \mathrm{H} & 13.351675 & -0.668067 & -2.300392 \\ \mathrm{H} & 14.439338 & -1.805937 & -1.483967 \\ \mathrm{H} & 12.745556 & -2.246461 & -1.774677 \\ \mathrm{O} & 14.589700 & 2.096929 & 1.684981 \\ \mathrm{H} & 14.231170 & 2.730404 & 2.320853 \\ \mathrm{O} & -14.868004 & -2.632585 & 0.115641 \\ \mathrm{H} & -15.403590 & -2.610990 & -0.688437\end{array}$

TABLE S4: Optimized x, y, z coordinates at B3LYP/6-31G (d, p) level for \#Zea ${ }^{\circ}(9)$.

$\begin{array}{lrrr}\text { C } & 11.580603 & -0.127843 & 0.224039 \\ \text { C } & 11.715071 & 0.974422 & 1.006209 \\ \text { C } & 12.676831 & -0.571073 & -0.775514 \\ \text { C } & 13.973246 & 0.248667 & -0.578412 \\ \text { H } & 14.628613 & 0.093332 & -1.446951 \\ \text { H } & 14.519253 & -0.120750 & 0.299225 \\ \text { C } & 13.715389 & 1.735286 & -0.364833 \\ \text { H } & 13.148180 & 2.140214 & -1.218241 \\ \mathrm{C} & 12.897354 & 1.914558 & 0.902725 \\ \mathrm{H} & 13.567362 & 1.768981 & 1.763911 \\ \mathrm{H} & 12.547185 & 2.952544 & 0.977969 \\ \mathrm{C} & 13.044846 & -2.060342 & -0.569290 \\ \mathrm{H} & 12.238228 & -2.735718 & -0.868096 \\ \mathrm{H} & 13.918167 & -2.312460 & -1.181005 \\ \mathrm{H} & 13.296590 & -2.266359 & 0.476328\end{array}$




\begin{tabular}{|c|c|c|c|}
\hline $\mathrm{C}$ & 12.151800 & -0.397643 & -2.221429 \\
\hline $\mathrm{H}$ & 12.882292 & -0.777337 & -2.945457 \\
\hline $\mathrm{H}$ & 11.216329 & -0.946755 & -2.364123 \\
\hline $\mathrm{H}$ & 11.953033 & 0.652216 & -2.456991 \\
\hline & 10.751931 & 1.374336 & 2.098735 \\
\hline $\mathrm{H}$ & 10.145400 & 2.241994 & 1.805577 \\
\hline & 10.072147 & 0.570090 & 2.380503 \\
\hline & 11.315128 & 1.683707 & 2.988670 \\
\hline & 10.384467 & -0.987106 & 0.256734 \\
\hline $\mathrm{H}$ & 10.560953 & -2.059223 & 0.225868 \\
\hline & 9.096142 & -0.557837 & 0.250152 \\
\hline $\mathrm{H}$ & 8.912126 & 0.514053 & 0.207498 \\
\hline & 7.912960 & -1.393124 & 0.268887 \\
\hline C & 6.682457 & -0.778412 & 0.220324 \\
\hline & 6.687975 & 0.310889 & 0.169800 \\
\hline $\mathrm{C}$ & 5.392723 & -1.381101 & 0.226838 \\
\hline & 5.326176 & -2.463572 & 0.282990 \\
\hline$C$ & 4.225631 & -0.650944 & 0.162892 \\
\hline 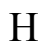 & 4.319968 & 0.433575 & 0.103394 \\
\hline & 2.898771 & -1.165578 & 0.163608 \\
\hline & 1.837387 & -0.260906 & 0.080743 \\
\hline $\mathrm{r}$ & 2.114339 & 0.792047 & 0.018840 \\
\hline 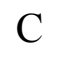 & 0.463444 & -0.532068 & 0.064416 \\
\hline $\mathrm{H}$ & 0.121668 & -1.563015 & 0.126988 \\
\hline $\mathrm{C}$ & 8.086316 & -2.889979 & 0.337803 \\
\hline $\mathrm{H}$ & 7.135919 & -3.423560 & 0.360820 \\
\hline $\mathrm{H}$ & 8.651907 & -3.257335 & -0.527148 \\
\hline 11 & 8.652521 & -3.176614 & 1.231728 \\
\hline $\mathrm{C}$ & 2.687699 & -2.658351 & 0.251087 \\
\hline $\mathrm{H}$ & 3.169709 & -3.171578 & -0.589232 \\
\hline $\mathrm{H}$ & 3.129700 & -3.062806 & 1.169137 \\
\hline 11 & 1.633365 & -2.935243 & 0.243741 \\
\hline $\mathrm{C}$ & -0.519535 & 0.461304 & -0.031342 \\
\hline $\mathrm{H}$ & -0.173095 & 1.490823 & -0.096686 \\
\hline $\mathrm{C}$ & -1.884746 & 0.198143 & -0.046182 \\
\hline 11 & -2.174034 & -0.850425 & 0.027487 \\
\hline a & -2.956709 & 1.121931 & -0.144239 \\
\hline $\mathrm{C}$ & -4.256521 & 0.627688 & -0.130397 \\
\hline $\mathrm{H}$ & -4.376349 & -0.451637 & -0.040821 \\
\hline $\mathrm{C}$ & -5.451108 & 1.390334 & -0.220237 \\
\hline $\mathrm{H}$ & -5.360732 & 2.470072 & -0.314225 \\
\hline $\mathrm{C}$ & -6.704357 & 0.842152 & -0.177689 \\
\hline $\mathrm{H}$ & -6.775208 & -0.234833 & -0.027221 \\
\hline . & -7.977061 & 1.550808 & -0.294433 \\
\hline $\mathrm{C}$ & -9.170380 & 0.786123 & 0.130171 \\
\hline $\mathrm{H}$ & -9.002270 & 0.032132 & 0.897392 \\
\hline
\end{tabular}




$\begin{array}{cccc}\mathrm{C} & -2.710883 & 2.611770 & -0.261756 \\ \mathrm{H} & -3.156368 & 3.151761 & 0.580908 \\ \mathrm{H} & -1.649729 & 2.859877 & -0.284253 \\ \mathrm{H} & -3.159173 & 3.013026 & -1.177085 \\ \mathrm{C} & -10.405721 & 0.917680 & -0.389059 \\ \mathrm{H} & -10.516063 & 1.589790 & -1.240381 \\ \mathrm{C} & -11.617524 & 0.173849 & 0.009704 \\ \mathrm{C} & -12.010910 & 0.038692 & 1.300620 \\ \mathrm{C} & -13.244400 & -0.754454 & 1.691209 \\ \mathrm{H} & -13.750122 & -0.249531 & 2.527091 \\ \mathrm{H} & -12.940314 & -1.739083 & 2.078732 \\ \mathrm{C} & -14.226381 & -0.986509 & 0.545392 \\ \mathrm{H} & -14.747022 & -0.041425 & 0.323869 \\ \mathrm{C} & -13.461053 & -1.443416 & -0.683608 \\ \mathrm{H} & -14.164620 & -1.678966 & -1.490281 \\ \mathrm{H} & -12.948110 & -2.379243 & -0.426927 \\ \mathrm{C} & -12.430161 & -0.402595 & -1.174191 \\ \mathrm{C} & -8.082539 & 2.821229 & -0.759675 \\ \mathrm{H} & -9.031549 & 3.345190 & -0.751963 \\ \mathrm{H} & -7.228761 & 3.361049 & -1.154438 \\ \mathrm{C} & -11.296863 & 0.645839 & 2.485014 \\ \mathrm{H} & -10.810433 & -0.128690 & 3.093760 \\ \mathrm{H} & -10.535438 & 1.370657 & 2.198388 \\ \mathrm{H} & -12.020215 & 1.142076 & 3.144272 \\ \mathrm{C} & -13.154874 & 0.746300 & -1.919704 \\ \mathrm{H} & -12.447263 & 1.484018 & -2.310536 \\ \mathrm{H} & -13.715574 & 0.348380 & -2.773026 \\ \mathrm{H} & -13.856720 & 1.275199 & -1.268547 \\ \mathrm{C} & -11.491032 & -1.117520 & -2.172418 \\ \mathrm{H} & -10.905466 & -1.895357 & -1.671914 \\ \mathrm{H} & -12.079244 & -1.593370 & -2.965158 \\ \mathrm{H} & -10.789439 & -0.425394 & -2.646167 \\ \mathrm{O} & -15.181485 & -2.000956 & 0.858455 \\ \mathrm{H} & -15.676340 & -1.709552 & 1.635750 \\ \mathrm{O} & 14.925011 & 2.475463 & -0.193204 \\ \mathrm{H} & 15.443354 & 2.380214 & -1.003142\end{array}$

TABLE S5: Optimized $x, y, z$ coordinates at B3LYP/6-31G (d, p) level for \#Zea ${ }^{\bullet}(13)$.
C 11.614569
$0.129154 \quad 0.012886$
$\begin{array}{llll}\text { C } & 11.817839 & -1.061165 & -0.609798\end{array}$
C $\quad 12.636348 \quad 0.721508 \quad 1.014306$
C $\quad 13.954474 \quad-0.087684 \quad 1.007861$
$\mathrm{H} \quad 14.549028 \quad 0.192224 \quad 1.888886$
$\mathrm{H} \quad 14.551727 \quad 0.178080 \quad 0.125997$ 


\begin{tabular}{|c|c|c|c|}
\hline $\mathrm{C}$ & 13.733335 & -1.594895 & 0.972572 \\
\hline & 13.118112 & -1.900534 & 3851 \\
\hline & 13.002947 & -1.953734 & -0.310054 \\
\hline H & 13.725966 & -1.905550 & -1.138774 \\
\hline $\mathrm{H}$ & 12.672392 & -3.000161 & -0.272123 \\
\hline & 12.998270 & 2.179534 & 0.641668 \\
\hline $\mathrm{H}$ & 12.165377 & 2.870820 & 0.797144 \\
\hline & 13.824197 & 2.526193 & 1.272650 \\
\hline & 13.319154 & 2.255072 & -0.402549 \\
\hline & 12.018731 & 0.723003 & 2.433796 \\
\hline & 12.700174 & 1.195644 & 3.150965 \\
\hline & 11.075490 & 1.277318 & 2.446718 \\
\hline & 11.805340 & -0.291378 & 2.784417 \\
\hline & 10.935766 & -1.620384 & -1.700951 \\
\hline 1 & 10.318005 & -2.451773 & -1.335078 \\
\hline & 10.270135 & -0.873554 & -2.134478 \\
\hline $\mathrm{H}$ & 11.561544 & -2.036036 & -2.501242 \\
\hline & 10.411011 & 0.948544 & -0.205518 \\
\hline 1 & 10.567287 & 2.021312 & -0.284767 \\
\hline & 9.129497 & 0.495045 & -0.239612 \\
\hline $\mathrm{H}$ & 8.955713 & -0.568284 & -0.085934 \\
\hline & 7.944227 & 1.299608 & -0.431777 \\
\hline & 6.714894 & 0.671754 & -0.395226 \\
\hline $\mathrm{H}$ & 6.728318 & -0.405092 & -0.223413 \\
\hline & 5.429225 & 1.249281 & -0.549556 \\
\hline $\mathrm{H}$ & 5.357046 & 2.318507 & -0.724964 \\
\hline & 4.258204 & 0.511897 & -0.481188 \\
\hline $\mathrm{H}$ & 4.356904 & -0.558779 & -0.300730 \\
\hline C & 2.941143 & & -0.614937 \\
\hline $\mathrm{C}$ & 1.870008 & 0.098813 & -0.497694 \\
\hline $\mathrm{H}$ & 2.150055 & -0.939423 & -0.316176 \\
\hline C & 0.509383 & 0.359538 & -0.578432 \\
\hline $\mathrm{H}$ & 0.163929 & 1.375636 & -0.755795 \\
\hline $\mathrm{C}$ & 8.103160 & 2.782944 & -0.656851 \\
\hline $\mathrm{H}$ & 7.149637 & 3.291587 & -0.799655 \\
\hline $\mathrm{H}$ & 8.606269 & 3.255749 & 0.195469 \\
\hline 11 & 8.722507 & 2.980132 & -1.539692 \\
\hline 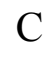 & 2.720429 & 2.479866 & -0.868774 \\
\hline $\mathrm{H}$ & 3.117396 & 3.084561 & -0.044822 \\
\hline $\mathrm{H}$ & 3.236332 & 2.801756 & -1.780697 \\
\hline 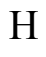 & 1.666076 & 2.731910 & -0.982872 \\
\hline & -0.486800 & -0.637616 & -0.433710 \\
\hline 1 & -0.140850 & -1.654727 & -0.253218 \\
\hline & -1.833944 & -0.390818 & -0.488258 \\
\hline & -2.143597 & 0.644220 & -0.629358 \\
\hline & -2.916232 & -1.361804 & -0.361413 \\
\hline
\end{tabular}




\begin{tabular}{|c|c|c|c|}
\hline $\mathrm{C}$ & -4.260954 & -0.782551 & -0 \\
\hline & -4.289871 & 0.254204 & 0.128926 \\
\hline & -5.442246 & -1.396620 & -0.455739 \\
\hline & -5.427821 & -2.413360 & -0.839820 \\
\hline & -6.718248 & -0.761280 & -0.249475 \\
\hline & -6.670411 & 0.260670 & 0.127306 \\
\hline & -7.962802 & -1.280847 & -0.460483 \\
\hline & -9.116720 & -0.441654 & -0.165735 \\
\hline & -8.885626 & 507 & 527 \\
\hline & -2.710800 & -2.707560 & -0.374418 \\
\hline & -1.731722 & 661 & -0 . \\
\hline & -3.520685 & -3.405964 & -0.198443 \\
\hline & -10.416472 & -0.801180 & -0.269661 \\
\hline 1 & -10.639866 & -1.827567 & -0.556576 \\
\hline & -11.585373 & 7733 & 203 \\
\hline 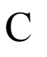 & -11.738250 & 1.306730 & -0.396867 \\
\hline & -12.930809 & 237 & -0.0 \\
\hline H & -13.225763 & 3074 & -0.882293 \\
\hline$\pi$ & -12.636141 & 2.879331 & 0.766564 \\
\hline 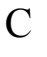 & -14.129776 & 225 & 91 \\
\hline $\mathrm{H}$ & -14.606280 & 0.852789 & -0.347951 \\
\hline & -13.652779 & 827 & 1.516886 \\
\hline H & -14.514033 & -0.172738 & 1.943686 \\
\hline $\mathrm{H}$ & -13.177294 & 0.903649 & 2.339309 \\
\hline C & -12.653806 & -0.667460 & 0.928776 \\
\hline $\mathrm{C}$ & -8.206661 & -2.67 & -0.973562 \\
\hline $\mathrm{H}$ & -8.800752 & -2.656177 & -1.894489 \\
\hline 1 & -7.28 & -3.216649 & \\
\hline $\mathrm{H}$ & -8.774122 & -3.268174 & -0.243412 \\
\hline 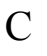 & -10.770840 & 2.007515 & -1.321350 \\
\hline $\mathrm{H}$ & -10.217989 & 2.798692 & -0.795948 \\
\hline $\mathrm{H}$ & -10.041232 & 1.330868 & -1.766096 \\
\hline $\mathrm{H}$ & -11.320314 & 2.505609 & -2.130098 \\
\hline $\mathrm{C}$ & -13.412358 & -1.715491 & 0.075853 \\
\hline $\mathrm{H}$ & -12.735530 & -2.475187 & -0.327529 \\
\hline $\mathrm{H}$ & -14.157326 & -2.235326 & 0.688883 \\
\hline $\mathrm{H}$ & -13.930171 & -1.254315 & -0.770064 \\
\hline 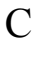 & -11.986971 & -1.394885 & 2.118572 \\
\hline $\mathrm{H}$ & -11.392954 & -0.699519 & 2.720143 \\
\hline 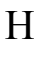 & -12.753569 & -1.834403 & 2.766651 \\
\hline 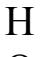 & -11.324386 & -2.200726 & 1.790761 \\
\hline $\mathrm{O}$ & -15.085322 & 2.212909 & 1.147085 \\
\hline & -15.395763 & 2.859917 & 0.499764 \\
\hline $\mathrm{O}$ & 14.962209 & -2.322739 & 0.972814 \\
\hline $\mathrm{H}$ & 15.42414 & -2.117766 & 1.79 \\
\hline
\end{tabular}


TABLE S6: Optimized $\mathrm{x}, \mathrm{y}, \mathrm{z}$ coordinates at B3LYP/6-31G (d, p) level for $\mathrm{Vio}^{\bullet+}$.

$\begin{array}{cccc}\mathrm{C} & -11.570939 & 0.010475 & 0.036163 \\ \mathrm{C} & -11.931772 & -0.731964 & 1.301103 \\ \mathrm{C} & -12.625607 & 0.264105 & -1.087497 \\ \mathrm{C} & -14.040541 & -0.196712 & -0.652578 \\ \mathrm{H} & -14.655074 & -0.318104 & -1.554324 \\ \mathrm{H} & -14.518589 & 0.590632 & -0.055560 \\ \mathrm{C} & -14.087566 & -1.475116 & 0.183648 \\ \mathrm{H} & -13.614797 & -2.305328 & -0.359193 \\ \mathrm{C} & -13.343584 & -1.250218 & 1.493529 \\ \mathrm{H} & -13.916243 & -0.536972 & 2.099569 \\ \mathrm{H} & -13.301777 & -2.186084 & 2.063699 \\ \mathrm{C} & -12.723229 & 1.763528 & -1.442117 \\ \mathrm{H} & -11.810851 & 2.138055 & -1.916916 \\ \mathrm{H} & -13.543671 & 1.915556 & -2.150132 \\ \mathrm{H} & -12.936084 & 2.379464 & -0.561011 \\ \mathrm{C} & -12.161099 & -0.511391 & -2.339506 \\ \mathrm{H} & -12.842115 & -0.318261 & -3.174672 \\ \mathrm{H} & -11.157336 & -0.198584 & -2.646479 \\ \mathrm{H} & -12.132277 & -1.587621 & -2.156315 \\ \mathrm{C} & -11.161156 & -0.547092 & 2.590942 \\ \mathrm{H} & -11.082174 & -1.509099 & 3.107918 \\ \mathrm{H} & -10.154724 & -0.165597 & 2.419141 \\ \mathrm{H} & -11.690563 & 0.143653 & 3.255405 \\ \mathrm{C} & -10.402670 & 0.931688 & 0.039757 \\ \mathrm{H} & -10.625675 & 1.993554 & 0.066639 \\ \mathrm{C} & -9.116526 & 0.505536 & -0.007971 \\ \mathrm{H} & -8.951093 & -0.568540 & -0.038710 \\ \mathrm{C} & -7.947952 & 1.352359 & -0.013710 \\ \mathrm{C} & -6.710087 & 0.730295 & -0.060322 \\ \mathrm{H} & -6.714206 & -0.358170 & -0.091350 \\ \mathrm{C} & -5.441033 & 1.348026 & -0.064698 \\ \mathrm{H} & -5.386250 & 2.431757 & -0.035121 \\ \mathrm{C} & -4.258138 & 0.626442 & -0.098602 \\ \mathrm{H} & -4.336747 & -0.459206 & -0.126086 \\ \mathrm{C} & -2.952410 & 1.170412 & -0.093191 \\ \mathrm{C} & -1.877562 & 0.268727 & -0.115978 \\ \mathrm{H} & -2.138808 & -0.788370 & -0.143534 \\ \mathrm{C} & -0.513519 & 0.568193 & -0.101447 \\ \mathrm{H} & -0.190196 & 1.605646 & -0.071649 \\ \mathrm{C} & -8.119948 & 2.847731 & 0.034113 \\ \mathrm{H} & -7.174709 & 3.388829 & 0.010720 \\ \mathrm{H} & -8.721393 & 3.191619 & -0.814355 \\ \mathrm{H} & -8.651697 & 3.144241 & 0.944855\end{array}$




\begin{tabular}{|c|c|c|c|}
\hline C. & -2.756485 & 2.666235 & -0.055512 \\
\hline 1 & -3.263407 & 3.144876 & -0.899733 \\
\hline & -3.180114 & 3.089460 & 0.861704 \\
\hline & -1.707559 & 2.956768 & -0.097168 \\
\hline & 0.479107 & -0.414308 & -0.119170 \\
\hline 1 & 0.154007 & -1.451176 & -0.149255 \\
\hline 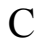 & 1.843966 & -0.119022 & -0.097168 \\
\hline & 2.109457 & 0.936892 & -0.065493 \\
\hline$C$ & 2.914364 & -1.026155 & -0.109142 \\
\hline C & 4.223550 & -0.490992 & -0.076176 \\
\hline H & 4.309863 & 0.593881 & -0.04 \\
\hline & 5.400102 & -1.223330 & -0.082189 \\
\hline $\mathrm{H}$ & 5.334743 & -2.306226 & 138 \\
\hline $\mathrm{C}$ & 6.676157 & -0.621227 & -0.042391 \\
\hline $\mathrm{H}$ & 6.693798 & 0.466842 & 527 \\
\hline $\mathrm{C}$ & 7.905734 & -1.260901 & 995 \\
\hline$C$ & 9.087200 & -0.433305 & 0973 \\
\hline$\pi$ & 8.938642 & 0.642 & 94 \\
\hline$C$ & 2.710877 & -2.520872 & -0.154778 \\
\hline $\mathrm{H}$ & 3.171408 & -3.001374 & 4836 \\
\hline $\mathrm{H}$ & 1.659560 & -2.805144 & -0.167087 \\
\hline $\mathrm{H}$ & 3.177756 & -2.947988 & -1.0 \\
\hline $\mathrm{C}$ & 10.366395 & -0.880614 & -0.023126 \\
\hline $\mathrm{H}$ & 10.573053 & -1.944522 & -0. \\
\hline $\mathrm{C}$ & 11.551597 & 0.018312 & 519 \\
\hline $\mathrm{C}$ & 11.974473 & 0.667535 & 1.295659 \\
\hline $\mathrm{C}$ & 13.413685 & 1.139628 & 1.489417 \\
\hline $\mathrm{H}$ & 13.758745 & 0.843557 & 2.487456 \\
\hline $\mathrm{H}$ & 13.402524 & 2.236066 & 1.464719 \\
\hline $\mathrm{C}$ & 14.407047 & 0.655314 & 0.425442 \\
\hline $\mathrm{H}$ & 14.680566 & -0.389871 & 0.640347 \\
\hline $\mathrm{C}$ & 13.786894 & 0.734851 & -0.961966 \\
\hline $\mathrm{H}$ & 14.549598 & 0.483984 & -1.706588 \\
\hline $\mathrm{H}$ & 13.485178 & 1.771269 & -1.145979 \\
\hline $\mathrm{C}$ & 12.573565 & -0.205852 & -1.146676 \\
\hline $\mathrm{C}$ & 8.057906 & -2.758178 & -0.105915 \\
\hline I & 8.622542 & -3.120628 & 0.759809 \\
\hline $\mathrm{H}$ & 7.105135 & -3.285964 & -0.125337 \\
\hline O & 11.214375 & 0.450048 & 2.587091 \\
\hline $\mathrm{H}$ & 11.238875 & 1.369736 & 3.181705 \\
\hline 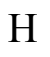 & 10.172394 & 0.182640 & 2.412054 \\
\hline 11 & 11.685610 & -0.340610 & 3.180520 \\
\hline 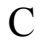 & 13.037119 & -1.680346 & -1.177280 \\
\hline $\mathrm{H}$ & 12.238081 & -2.352403 & -1.504690 \\
\hline $\mathrm{H}$ & 13.860669 & -1.794392 & -1.888681 \\
\hline & 13.391285 & -2.030667 & -0.202665 \\
\hline
\end{tabular}




$\begin{array}{lrrr}\mathrm{C} & 11.890718 & 0.142834 & -2.484999 \\ \mathrm{H} & 11.515819 & 1.170440 & -2.472191 \\ \mathrm{H} & 12.605398 & 0.052584 & -3.309395 \\ \mathrm{H} & 11.049574 & -0.525483 & -2.696203 \\ \mathrm{O} & 15.578384 & 1.464500 & 0.406354 \\ \mathrm{H} & 16.024192 & 1.375160 & 1.259187 \\ \mathrm{O} & -15.425400 & -1.811623 & 0.542115 \\ \mathrm{H} & -15.895803 & -2.070335 & -0.261158 \\ \mathrm{O} & 11.249931 & 1.393882 & 0.276280 \\ \mathrm{O} & -11.211845 & -1.372959 & 0.228193 \\ \mathrm{H} & 8.618836 & -3.051478 & -1.000161\end{array}$

TABLE S7: Optimized x, y, z coordinates at B3LYP/6-31G (d, p) level for \#Vio (4).

$\begin{array}{lccc}\mathrm{C} & 11.560172 & 0.028238 & 0.028318 \\ \mathrm{C} & 12.079428 & -0.487815 & -1.283655 \\ \mathrm{C} & 12.465353 & 0.053462 & 1.298186 \\ \mathrm{C} & 13.921377 & -0.375640 & 0.976206 \\ \mathrm{H} & 14.408690 & -0.679507 & 1.913207 \\ \mathrm{H} & 14.485986 & 0.487916 & 0.600516 \\ \mathrm{C} & 14.065600 & -1.482114 & -0.067188 \\ \mathrm{H} & 13.515042 & -2.378763 & 0.248552 \\ \mathrm{C} & 13.503533 & -1.000403 & -1.397553 \\ \mathrm{H} & 14.153049 & -0.203156 & -1.781748 \\ \mathrm{H} & 13.534927 & -1.814741 & -2.131835 \\ \mathrm{C} & 12.535002 & 1.468752 & 1.911108 \\ \mathrm{H} & 11.569874 & 1.790788 & 2.311921 \\ \mathrm{H} & 13.254816 & 1.474158 & 2.736536 \\ \mathrm{H} & 12.869953 & 2.212535 & 1.179240 \\ \mathrm{C} & 11.834964 & -0.900845 & 2.335050 \\ \mathrm{H} & 12.396120 & -0.864064 & 3.275236 \\ \mathrm{H} & 10.800007 & -0.613757 & 2.546971 \\ \mathrm{H} & 11.825694 & -1.931946 & 1.974221 \\ \mathrm{C} & 11.464578 & -0.069012 & -2.601932 \\ \mathrm{H} & 11.430345 & -0.927238 & -3.281922 \\ \mathrm{H} & 10.451529 & 0.310862 & -2.470490 \\ \mathrm{H} & 12.073481 & 0.708109 & -3.077053 \\ \mathrm{C} & 10.389442 & 0.958534 & 0.053668 \\ \mathrm{H} & 10.618889 & 2.014321 & 0.163553 \\ \mathrm{C} & 9.109023 & 0.545061 & -0.030535 \\ \mathrm{H} & 8.938667 & -0.524041 & -0.140641 \\ \mathrm{C} & 7.922816 & 1.389093 & 0.002643 \\ \mathrm{C} & 6.700573 & 0.782139 & -0.071507 \\ \mathrm{H} & 6.700675 & -0.305346 & -0.148606 \\ \mathrm{C} & 5.401324 & 1.393144 & -0.058616\end{array}$




$\begin{array}{lrrr}\mathrm{H} & 5.341293 & 2.475851 & 0.002872 \\ \mathrm{C} & 4.246179 & 0.671178 & -0.119024 \\ \mathrm{H} & 4.332787 & -0.414115 & -0.175419 \\ \mathrm{C} & 2.900543 & 1.193099 & -0.112761 \\ \mathrm{C} & 1.857822 & 0.300952 & -0.160402 \\ \mathrm{H} & 2.123879 & -0.755691 & -0.199251 \\ \mathrm{C} & 0.455983 & 0.576436 & -0.160863 \\ \mathrm{H} & 0.124953 & 1.612554 & -0.132125 \\ \mathrm{C} & 8.108934 & 2.880861 & 0.122509 \\ \mathrm{H} & 7.164496 & 3.425623 & 0.127032 \\ \mathrm{H} & 8.643031 & 3.133587 & 1.046632 \\ \mathrm{H} & 8.712858 & 3.266321 & -0.707732 \\ \mathrm{C} & 2.708170 & 2.688921 & -0.049748 \\ \mathrm{H} & 3.155358 & 3.100803 & 0.862685 \\ \mathrm{H} & 3.200801 & 3.180601 & -0.896717 \\ \mathrm{H} & 1.657726 & 2.979949 & -0.063344 \\ \mathrm{C} & -0.503392 & -0.398247 & -0.191135 \\ \mathrm{H} & -0.170023 & -1.433687 & -0.215784 \\ \mathrm{C} & -1.906180 & -0.128456 & -0.186488 \\ \mathrm{H} & -2.178175 & 0.927252 & -0.170020 \\ \mathrm{C} & -2.943182 & -1.028835 & -0.193888 \\ \mathrm{C} & -4.293081 & -0.518843 & -0.179141 \\ \mathrm{H} & -4.390535 & 0.566969 & -0.177529 \\ \mathrm{C} & -5.440076 & -1.256582 & -0.160590 \\ \mathrm{H} & -5.365376 & -2.340020 & -0.156180 \\ \mathrm{C} & -6.747830 & -0.665977 & -0.140074 \\ \mathrm{H} & -6.766877 & 0.423915 & -0.157391 \\ \mathrm{C} & -1.959305 & -1.298566 & -0.094681 \\ \mathrm{C} & -13.237653 & 1.79359 \\ \mathrm{H} & -9.015601 & -0.479326 & -0.070869 \\ \mathrm{C} & -2.739619 & -2.524612 & -0.210132 \\ \mathrm{H} & -3.222072 & -2.973498 & -1.086202 \\ \mathrm{H} & -1.687054 & -2.807111 & -0.231038 \\ \mathrm{H} & -3.190179 & -2.988369 & 0.675331 \\ \mathrm{C} & -10.431380 & -0.926587 & 0.016231 \\ \mathrm{H} & -10.635666 & -1.991421 & 0.085325 \\ \mathrm{C} & -11.621514 & -0.033098 & 0.062508 \\ \mathrm{C} & -12.172639 & 0.572299 & -1.215242 \\ \mathrm{H} & -13.597245 & 0.885071 & -1.277251 \\ \mathrm{H} & -14.049345 & 1.010920 & -2.259593 \\ \mathrm{H} & -0.273116 & 1.239361\end{array}$




$\begin{array}{rrrr}\mathrm{C} & -8.116962 & -2.798125 & -0.056666 \\ \mathrm{H} & -8.731022 & -3.146270 & -0.895831 \\ \mathrm{H} & -7.163705 & -3.325183 & -0.101830 \\ \mathrm{H} & -8.627475 & -3.112686 & 0.861814 \\ \mathrm{C} & -11.480454 & 0.386447 & -2.547823 \\ \mathrm{H} & -10.433923 & 0.112239 & -2.419502 \\ \mathrm{H} & -11.978091 & -0.394540 & -3.132750 \\ \mathrm{C} & -13.233016 & -1.679494 & 1.127919 \\ \mathrm{H} & -12.486374 & -2.467812 & 1.263753 \\ \mathrm{H} & -13.991836 & -1.816076 & 1.905959 \\ \mathrm{H} & -13.714596 & -1.839972 & 0.157968 \\ \mathrm{C} & -11.837767 & -0.153576 & 2.570966 \\ \mathrm{H} & -11.347995 & 0.822158 & 2.648600 \\ \mathrm{H} & -12.524843 & -0.256684 & 3.417966 \\ \mathrm{H} & -11.067758 & -0.924999 & 2.663195 \\ \mathrm{O} & -15.281859 & 2.101126 & 0.001282 \\ \mathrm{H} & -15.799508 & 2.168770 & -0.812126 \\ \mathrm{O} & 15.439632 & -1.801289 & -0.299070 \\ \mathrm{H} & 15.793691 & -2.177640 & 0.517316 \\ \mathrm{O} & -11.380113 & 1.342438 & -0.231140 \\ \mathrm{O} & 11.238563 & -1.302130 & -0.437333 \\ \mathrm{H} & -11.528314 & 1.318648 & -3.120575\end{array}$

TABLE S8: Optimized $\mathrm{x}, \mathrm{y}, \mathrm{z}$ coordinates at B3LYP/6-31G (d, p) level for \#Vio ${ }^{\circ}(5)$.
C $\quad-11.574732-0.001844 \quad 0.069182$
C $\quad-11.950482-0.750390 \quad 1.317933$
$\begin{array}{llll}\text { C } & -12.616577 & 0.237627 & -1.068035\end{array}$
C $\quad-14.031623-0.251428-0.662630$
$\mathrm{H} \quad-14.628950-0.382602-1.575698$
H $\quad-14.535591 \quad 0.525505 \quad-0.072634$
$\begin{array}{llll}\text { C } & -14.070077 & -1.530730 & 0.170898\end{array}$
$\mathrm{H} \quad-13.568557 \quad-2.349572 \quad-0.362952$
C $\quad-13.356891 \quad-1.293613 \quad 1.494703$
H $\quad-13.952309-0.588253 \quad 2.088802$
H $\quad-13.311744 \quad-2.228816 \quad 2.066365$
C $\quad-12.737161 \quad 1.737206 \quad-1.414206$
$\mathrm{H} \quad-11.818262 \quad 2.131686-1.856823$
$\mathrm{H} \quad-13.543118 \quad 1.880206-2.141878$
$\mathrm{H} \quad-12.981693 \quad 2.339551 \quad-0.531889$
C $\quad-12.119078 \quad-0.517290 \quad-2.319213$
$\mathrm{H} \quad-12.782846 \quad-0.322567-3.168786$
H $\quad-11.111471-0.189872-2.594977$

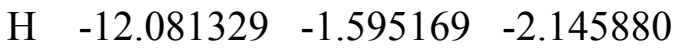
C $\quad-11.192223 \quad-0.566206 \quad 2.614969$ 


$\begin{array}{cccc}\mathrm{H} & -11.086533 & -1.532481 & 3.120232 \\ \mathrm{H} & -10.198468 & -0.152366 & 2.445187 \\ \mathrm{H} & -11.742130 & 0.102096 & 3.286812 \\ \mathrm{C} & -10.407893 & 0.932835 & 0.081655 \\ \mathrm{H} & -10.642856 & 1.990063 & 0.159532 \\ \mathrm{C} & -9.125658 & 0.526080 & -0.008906 \\ \mathrm{H} & -8.948336 & -0.545190 & -0.079290 \\ \mathrm{C} & -7.945399 & 1.378978 & -0.005537 \\ \mathrm{C} & -6.719242 & 0.779277 & -0.074414 \\ \mathrm{H} & -6.711891 & -0.309452 & -0.130835 \\ \mathrm{C} & -5.424218 & 1.399367 & -0.071873 \\ \mathrm{H} & -5.371576 & 2.483120 & -0.023740 \\ \mathrm{C} & -4.264047 & 0.684548 & -0.118686 \\ \mathrm{H} & -4.343092 & -0.401883 & -0.162962 \\ \mathrm{C} & -2.921886 & 1.215407 & -0.107195 \\ \mathrm{C} & -1.873134 & 0.329823 & -0.139939 \\ \mathrm{H} & -2.132088 & -0.728611 & -0.177885 \\ \mathrm{C} & -0.472958 & 0.613828 & -0.123941 \\ \mathrm{H} & -0.148403 & 1.651692 & -0.085971 \\ \mathrm{C} & -8.141256 & 2.871752 & 0.082010 \\ \mathrm{H} & -7.200881 & 3.423163 & 0.062890 \\ \mathrm{H} & -8.756510 & 3.232927 & -0.750883 \\ \mathrm{H} & -8.667579 & 3.142622 & 1.005394 \\ \mathrm{C} & -2.739657 & 2.712854 & -0.052977 \\ \mathrm{H} & -3.230059 & 3.195302 & -0.906531 \\ \mathrm{H} & -3.195298 & 3.128288 & 0.853635 \\ \mathrm{H} & -1.691037 & 3.010546 & -0.062689 \\ \mathrm{C} & 0.491972 & -0.355326 & -0.148713 \\ \mathrm{H} & 0.164233 & -1.392165 & -0.185859 \\ \mathrm{C} & 1.893483 & -0.079143 & -0.124062 \\ \mathrm{H} & 2.160553 & 0.977248 & -0.085693 \\ \mathrm{C} & 2.934095 & -0.974982 & -0.139310 \\ \mathrm{C} & 4.282295 & -0.460782 & -0.101349 \\ \mathrm{H} & 4.376023 & 0.624668 & -0.062519 \\ \mathrm{C} & 5.430994 & -1.195520 & -0.107503 \\ \mathrm{H} & 5.358643 & -2.278354 & -0.146889 \\ \mathrm{C} & 6.738368 & -0.604392 & -0.061930 \\ \mathrm{H} & 6.756324 & 0.484818 & -0.019144 \\ \mathrm{C} & 7.949980 & -1.237404 & -0.064915 \\ \mathrm{H} & 9.153392 & -0.420223 & -0.009741 \\ \mathrm{H} & 3.736722 & -2.470811 & -0.191525 \\ \mathrm{H} & -2.954311 & 0.679621 \\ \mathrm{H} & -2.65314 & -2.757329 & -0.214129 \\ \mathrm{H} & -0.871885 & -0.016702\end{array}$




$\begin{array}{cccc}\mathrm{H} & 10.627203 & -1.937075 & -0.069405 \\ \mathrm{C} & 11.618574 & 0.023289 & 0.026211 \\ \mathrm{C} & 12.067298 & 0.621061 & 1.361630 \\ \mathrm{C} & 13.507865 & 1.090454 & 1.550905 \\ \mathrm{H} & 13.853738 & 0.793320 & 2.548636 \\ \mathrm{H} & 13.512607 & 2.186441 & 1.518556 \\ \mathrm{C} & 14.490164 & 0.585142 & 0.490656 \\ \mathrm{H} & 14.718557 & -0.474305 & 0.688901 \\ \mathrm{C} & 13.887739 & 0.716055 & -0.899610 \\ \mathrm{H} & 14.650532 & 0.464383 & -1.644581 \\ \mathrm{H} & 13.614748 & 1.764752 & -1.058493 \\ \mathrm{C} & 12.650790 & -0.186808 & -1.114855 \\ \mathrm{C} & 8.108615 & -2.736133 & -0.125250 \\ \mathrm{H} & 8.661411 & -3.104711 & 0.747226 \\ \mathrm{H} & 7.154267 & -3.261966 & -0.159588 \\ \mathrm{H} & 8.683021 & -3.030802 & -1.011949 \\ \mathrm{C} & 11.313652 & 0.363567 & 2.577601 \\ \mathrm{H} & 10.267511 & 0.093555 & 2.532080 \\ \mathrm{H} & 11.790130 & 0.454285 & 3.546262 \\ \mathrm{C} & 13.078282 & -1.669737 & -1.194011 \\ \mathrm{H} & 12.259187 & -2.306306 & -1.539772 \\ \mathrm{H} & 13.900427 & -1.782723 & -1.908277 \\ \mathrm{H} & 13.419144 & -2.060087 & -0.229820 \\ \mathrm{C} & 11.984050 & 0.220433 & -2.445323 \\ \mathrm{H} & 11.641945 & 1.258716 & -2.401940 \\ \mathrm{H} & 12.698432 & 0.130638 & -3.271061 \\ \mathrm{H} & 11.120911 & -0.412472 & -2.671182 \\ \mathrm{O} & 15.699213 & 1.344100 & 0.502246 \\ \mathrm{H} & 16.090692 & 1.264514 & 1.382327 \\ \mathrm{O} & -15.412623 & -1.900313 & 0.494184 \\ \mathrm{H} & -15.860221 & -2.126394 & -0.331656 \\ \mathrm{O} & 11.341007 & 1.386063 & 0.324398 \\ \mathrm{O} & -11.213739 & -1.389748 & 0.253366\end{array}$

TABLE S9: Optimized x, y, z coordinates at B3LYP/6-31G (d, p) level for \#Vio ${ }^{\circ}(9)$.
C $\quad 11.555632 \quad 0.054174 \quad-0.019598$
C $\quad 12.062929-0.905224-1.059384$
$\begin{array}{llll}\text { C } & 12.479842 & 0.552057 & 1.135310\end{array}$
C $\quad 13.937503 \quad 0.051577 \quad 0.960126$
$\mathrm{H} \quad 14.447981 \quad 0.122535 \quad 1.930711$
H $\quad 14.477599 \quad 0.720480 \quad 0.277071$
C $\quad 14.082529-1.361575 \quad 0.399256$
H $\quad 13.550969-2.080898 \quad 1.037026$
C $\quad 13.491992-1.414047-1.002768$ 


\begin{tabular}{|c|c|c|c|}
\hline & 23972 & -0.813112 & -1.66 \\
\hline & 3.521978 & -2.443183 & \\
\hline & 12.533649 & 2.093889 & 89202 \\
\hline & 11.568622 & 2.529142 & 1463165 \\
\hline & 13.264086 & 2.407228 & 722 \\
\hline & 12.846433 & 2.5 & 0.2 \\
\hline & 11.8 & 0.0 & \\
\hline & 12.457708 & 0.4 & \\
\hline & 10.8 & & \\
\hline & 4667 & -1.0 & \\
\hline & 11.4 & -1.0 & -2.4 \\
\hline & 11.389216 & -2.0 & -2.7 \\
\hline & 10. & -0.6 & \\
\hline & 12. & -0 . & \\
\hline & 10. & 0.9 & -0.3 \\
\hline & & & -0. \\
\hline & 9.0 & 0.4 & -0.2 \\
\hline & & -0.5 & \\
\hline $\mathrm{C}$ & 7.9 & 1.2 & -0.4 \\
\hline & 6.6 & 0.6 & -0.3 \\
\hline $\mathrm{H}$ & 6.6 & -0.3 & -0 \\
\hline & 5.3 & 1.2 & -0.4 \\
\hline $\mathrm{H}$ & & 2.3 & -0.7 \\
\hline & 4.2 & 0.5 & -0.2 \\
\hline $\mathrm{H}$ & & -0.4 & \\
\hline & 2.8 & 1.0 & -0.4 \\
\hline $\mathrm{C}$ & & 0.1 & -0.1 \\
\hline $\mathrm{H}$ & 2.1 & -0.8 & 0.1 \\
\hline $\mathrm{C}$ & 0.45 & 0.4 & -0.2 \\
\hline $\mathrm{H}$ & & 1.4 & -0.5 \\
\hline & & 2.7 & -0.8 \\
\hline $\mathrm{H}$ & 7.1 & 3.2 & -1.0 \\
\hline $\mathrm{H}$ & 8.6 & 3.2 & -0.1 \\
\hline $\mathrm{H}$ & 8.6 & 2.8 & -1.8 \\
\hline $\mathrm{C}$ & 2.68 & 2.4 & -0.8 \\
\hline $\mathrm{H}$ & 3.15 & 3.1 & -0.12 \\
\hline $\mathrm{H}$ & 3.132180 & 2.676951 & -1.81 \\
\hline $\mathrm{H}$ & 1.6 & 2.7 & -0.9 \\
\hline C & -0.5 & -0.5 & 0.050822 \\
\hline $\mathrm{H}$ & -0.18 & -1.505 & 0.339472 \\
\hline $\mathrm{C}$ & -1.892822 & -0.258053 & -0.019728 \\
\hline 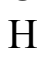 & -2.17 & 0.752403 & -0.313645 \\
\hline C & -2.965529 & -1.144355 & 0.240242 \\
\hline $\mathrm{C}$ & -4.266227 & -0.664097 & 0.102563 \\
\hline $\mathrm{H}$ & -4.380333 & 0.377401 & -0.196733 \\
\hline $\mathrm{C}$ & -5.462239 & -1.395438 & 0.309495 \\
\hline
\end{tabular}




$\begin{array}{lrrr}\mathrm{H} & -5.375547 & -2.438257 & 0.605417 \\ \mathrm{C} & -6.712478 & -0.857974 & 0.143896 \\ \mathrm{H} & -6.766655 & 0.181737 & -0.176696 \\ \mathrm{C} & -7.996946 & -1.521392 & 0.340250 \\ \mathrm{C} & -9.179094 & -0.721982 & -0.055119 \\ \mathrm{H} & -9.022772 & 0.025327 & -0.831025 \\ \mathrm{C} & -2.726494 & -2.581002 & 0.655394 \\ \mathrm{H} & -3.162166 & -3.277603 & -0.069326 \\ \mathrm{H} & -1.666471 & -2.819779 & 0.740794 \\ \mathrm{H} & -3.188442 & -2.792187 & 1.626016 \\ \mathrm{C} & -10.408631 & -0.817433 & 0.470363 \\ \mathrm{H} & -10.595609 & -1.511497 & 1.286245 \\ \mathrm{C} & -11.568217 & 0.020185 & 0.033795 \\ \mathrm{C} & -12.305901 & -0.312079 & -1.232605 \\ \mathrm{C} & -13.745743 & 0.153728 & -1.447032 \\ \mathrm{H} & -14.336758 & -0.673029 & -1.861306 \\ \mathrm{H} & -13.725671 & 0.945751 & -2.205869 \\ \mathrm{C} & -14.434368 & 0.712586 & -0.197718 \\ \mathrm{H} & -14.757924 & -0.127200 & 0.437839 \\ \mathrm{C} & -13.480996 & 1.596907 & 0.592022 \\ \mathrm{H} & -14.030125 & 2.064515 & 1.416758 \\ \mathrm{H} & -13.134721 & 2.402056 & -0.064335 \\ \mathrm{C} & -12.265453 & 0.827350 & 1.160498 \\ \mathrm{C} & -8.129321 & -2.779520 & 0.833478 \\ \mathrm{H} & -9.099266 & -3.259607 & 0.894823 \\ \mathrm{H} & -7.278624 & -3.358933 & 1.174468 \\ \mathrm{C} & -11.893657 & -1.473207 & -2.112982 \\ \mathrm{H} & -12.046844 & -1.208237 & -3.165312 \\ \mathrm{H} & -10.845653 & -1.735300 & -1.971034 \\ \mathrm{H} & -12.508878 & -2.354337 & -1.899025 \\ \mathrm{C} & -12.714432 & -0.115936 & 2.300032 \\ \mathrm{H} & -11.858591 & -0.531644 & 2.839289 \\ \mathrm{H} & -13.316805 & 0.438417 & 3.026969 \\ \mathrm{H} & -13.318538 & -0.953654 & 1.937033 \\ \mathrm{C} & -11.262916 & 1.856266 & 1.720830 \\ \mathrm{H} & -10.903017 & 2.514952 & 0.924824 \\ \mathrm{H} & -11.742769 & 2.476156 & 2.485971 \\ \mathrm{H} & -10.396679 & 1.365993 & 2.174669 \\ & -15.567539 & 1.510831 & -0.541471 \\ \mathrm{H} & -16.183067 & 0.953063 & -1.035569 \\ \mathrm{H} & 15.829856 & -1.778556 & 1.164023 \\ \mathrm{H} & 0.772031 & -1.173327 \\ \mathrm{H} & -1.356782 & 0.042025\end{array}$


TABLE S10: Optimized x, y, z coordinates at B3LYP/6-31G (d, p) level for \#Vio ${ }^{\circ}(13)$.

\begin{tabular}{|c|c|c|c|}
\hline $\mathrm{C}$ & -11.563080 & 0.089138 & 0.128628 \\
\hline $\mathrm{C}$ & -11.933927 & -0.592771 & 1.416719 \\
\hline $\mathrm{C}$ & -12.621990 & 0.313660 & -0.995516 \\
\hline $\mathrm{C}$ & -14.042458 & -0.109693 & -0.538584 \\
\hline $\mathrm{H}$ & -14.664677 & -0.258853 & -1.432041 \\
\hline $\mathrm{H}$ & -14.506506 & 0.708191 & 0.028396 \\
\hline $\mathrm{C}$ & -14.103553 & -1.350489 & 0.349794 \\
\hline $\mathrm{H}$ & -13.641907 & -2.207799 & -0.158958 \\
\hline $\mathrm{C}$ & -13.352606 & -1.081887 & 1.646514 \\
\hline $\mathrm{H}$ & -13.911509 & -0.333260 & 2.222872 \\
\hline $\mathrm{H}$ & -13.323787 & -1.993316 & 2.256303 \\
\hline $\mathrm{C}$ & -12.702309 & 1.800171 & -1.404510 \\
\hline $\mathrm{H}$ & -11.785926 & 2.141830 & -1.893859 \\
\hline $\mathrm{H}$ & -13.527130 & 1.940041 & -2.111282 \\
\hline $\mathrm{H}$ & -12.895754 & 2.448883 & -0.542655 \\
\hline $\mathrm{C}$ & -12.177203 & -0.510634 & -2.222541 \\
\hline $\mathrm{H}$ & -12.858525 & -0.337975 & -3.062910 \\
\hline $\mathrm{H}$ & -11.169727 & -0.222171 & -2.539312 \\
\hline $\mathrm{H}$ & -12.161263 & -1.579998 & -2.000176 \\
\hline $\mathrm{C}$ & -11.141187 & -0.376518 & 2.687971 \\
\hline $\mathrm{H}$ & -11.054593 & -1.322386 & 3.233857 \\
\hline $\mathrm{H}$ & -10.138916 & -0.003032 & 2.478992 \\
\hline $\mathrm{H}$ & -11.654322 & 0.338334 & 3.340710 \\
\hline $\mathrm{C}$ & -10.367755 & 0.984597 & 0.075210 \\
\hline $\mathrm{H}$ & -10.566893 & 2.051309 & 0.110178 \\
\hline $\mathrm{C}$ & -9.098353 & 0.531637 & -0.026453 \\
\hline $\mathrm{H}$ & -8.958401 & -0.547146 & -0.052994 \\
\hline $\mathrm{C}$ & -7.898392 & 1.343203 & -0.090282 \\
\hline $\mathrm{C}$ & -6.683547 & 0.699519 & -0.169286 \\
\hline $\mathrm{H}$ & -6.714898 & -0.390267 & -0.182697 \\
\hline $\mathrm{C}$ & -5.385422 & 1.275417 & -0.226743 \\
\hline $\mathrm{H}$ & -5.296206 & 2.357682 & -0.216417 \\
\hline $\mathrm{C}$ & -4.230105 & 0.518251 & -0.285394 \\
\hline $\mathrm{H}$ & -4.346809 & -0.565655 & -0.291163 \\
\hline $\mathrm{C}$ & -2.901130 & 1.007054 & -0.329479 \\
\hline $\mathrm{C}$ & -1.848881 & 0.074632 & -0.365838 \\
\hline $\mathrm{H}$ & -2.147009 & -0.974363 & -0.367835 \\
\hline $\mathrm{C}$ & -0.481030 & 0.321313 & -0.390716 \\
\hline $\mathrm{H}$ & -0.118209 & 1.346857 & -0.384845 \\
\hline $\mathrm{C}$ & -8.041058 & 2.843964 & -0.060675 \\
\hline $\mathrm{H}$ & -7.083986 & 3.361386 & -0.130811 \\
\hline $\mathrm{H}$ & -8.668544 & 3.191564 & -0.890292 \\
\hline $\mathrm{H}$ & -8.529862 & 3.171728 & 0.864919 \\
\hline $\mathrm{C}$ & -2.657040 & 2.497400 & -0.325666 \\
\hline
\end{tabular}




\begin{tabular}{|c|c|c|c|}
\hline $\mathrm{H}$ & -3.172886 & 2.980227 & -1.163553 \\
\hline $\mathrm{H}$ & -3.039947 & 2.955792 & 0.593851 \\
\hline $\mathrm{H}$ & -1.599423 & 2.749903 & -0.403195 \\
\hline $\mathrm{O}$ & 0.495924 & -0.701127 & -0.416192 \\
\hline $\mathrm{H}$ & 0.131786 & -1.727763 & -0.423179 \\
\hline $\mathrm{C}$ & 1.848280 & -0.466933 & -0.431038 \\
\hline $\mathrm{H}$ & 2.166465 & 0.575009 & -0.430458 \\
\hline $\mathrm{C}$ & 2.923007 & -1.451233 & -0.438101 \\
\hline $\mathrm{C}$ & 4.282434 & -0.886683 & -0.488739 \\
\hline $\mathrm{H}$ & 4.350769 & 0.138665 & -0.852418 \\
\hline $\mathrm{C}$ & 5.429885 & -1.494089 & -0.104886 \\
\hline $\mathrm{H}$ & 5.375408 & -2.497002 & 0.310804 \\
\hline $\mathrm{C}$ & 6.721302 & -0.860905 & -0.198475 \\
\hline $\mathrm{H}$ & 6.714468 & 0.145370 & -0.617492 \\
\hline $\mathrm{C}$ & 7.933457 & -1.361536 & 0.175517 \\
\hline $\mathrm{C}$ & 9.109196 & -0.518732 & -0.007727 \\
\hline $\mathrm{H}$ & 8.935980 & 0.467059 & -0.435047 \\
\hline $\mathrm{C}$ & 2.706587 & -2.795528 & -0.410072 \\
\hline $\mathrm{H}$ & 1.711232 & -3.217030 & -0.331633 \\
\hline $\mathrm{H}$ & 3.525339 & -3.501954 & -0.479450 \\
\hline $\mathrm{C}$ & 10.379101 & -0.836724 & 0.308996 \\
\hline $\mathrm{H}$ & 10.613173 & -1.808375 & 0.735064 \\
\hline $\mathrm{C}$ & 11.540751 & 0.078779 & 0.086995 \\
\hline $\mathrm{C}$ & 11.810267 & 1.200939 & 1.050253 \\
\hline $\mathrm{C}$ & 13.205607 & 1.811120 & 1.176572 \\
\hline $\mathrm{H}$ & 13.449457 & 1.943511 & 2.238476 \\
\hline $\mathrm{H}$ & 13.170188 & 2.811634 & 0.727834 \\
\hline $\mathrm{C}$ & 14.317226 & 1.019155 & 0.480302 \\
\hline $\mathrm{H}$ & 14.588717 & 0.156099 & 1.109161 \\
\hline $\mathrm{C}$ & 13.849398 & 0.518000 & -0.878007 \\
\hline $\mathrm{H}$ & 14.694946 & 0.052555 & -1.396443 \\
\hline $\mathrm{H}$ & 13.538839 & 1.381941 & -1.474797 \\
\hline $\mathrm{C}$ & 12.686326 & -0.497556 & -0.786209 \\
\hline $\mathrm{C}$ & 8.125568 & -2.734424 & 0.769581 \\
\hline $\mathrm{H}$ & 8.580341 & -2.666585 & 1.765177 \\
\hline $\mathrm{H}$ & 7.191105 & -3.286950 & 0.868874 \\
\hline $\mathrm{H}$ & 8.804175 & -3.334056 & 0.151010 \\
\hline $\mathrm{C}$ & 10.913551 & 1.453602 & 2.244107 \\
\hline $\mathrm{H}$ & 9.909172 & 1.064069 & 2.081769 \\
\hline $\mathrm{H}$ & 11.331027 & 0.985443 & 3.142535 \\
\hline $\mathrm{C}$ & 13.188254 & -1.837021 & -0.200845 \\
\hline $\mathrm{H}$ & 12.435480 & -2.624595 & -0.297789 \\
\hline $\mathrm{H}$ & 14.078953 & -2.170410 & -0.743378 \\
\hline $\mathrm{H}$ & 13.453435 & -1.758938 & 0.858519 \\
\hline $\mathrm{C}$ & 12.144557 & -0.739652 & -2.209581 \\
\hline $\mathrm{H}$ & 11.753509 & 0.188981 & -2.635879 \\
\hline
\end{tabular}




$\begin{array}{lrrr}\mathrm{H} & 12.943661 & -1.105441 & -2.863604 \\ \mathrm{H} & 11.338570 & -1.479406 & -2.211227 \\ \mathrm{O} & 15.473413 & 1.827666 & 0.259257 \\ \mathrm{H} & 15.781091 & 2.145088 & 1.118713 \\ \mathrm{O} & -15.449593 & -1.660257 & 0.718083 \\ \mathrm{H} & -15.923730 & -1.905118 & -0.087328 \\ \mathrm{O} & 11.189076 & 1.436181 & -0.235283 \\ \mathrm{O} & -11.241480 & -1.300452 & 0.365668 \\ \mathrm{H} & 10.844821 & 2.531094 & 2.431664\end{array}$

